\title{
Shrinking and scaling electrophysiology
}

\begin{abstract}
An in vivo alternative to patch-clamping aims to overcome the electrophysiology bottleneck in C. elegans and other small invertebrates
\end{abstract}

The go-to procedure for performing electrophysiology measurements in Caenorhabditis elegans is patch-clamping via manual dissection and a glass micropipette electrode. But for a model organism increasingly valued for its high throughput potential, patch-clamp recording is slownot to mention it can damage the worms. Overcoming this electrophysiology bottleneck has been a focus of Jacob Robinson's nano-neurotechnology lab at Rice University. He and his colleagues propose nanoscale suspended electrode arrays, or nanoSPEARs, as a scalable in vivo solution (Nat. Nanotechnol. 10.1038/nnano.2017.55; published online 17 April 2017).

As the name suggests, the technology is tiny-the nanowire electrodes developed for the platform are 25 times smaller than a single C. elegans muscle cell. The electrodes are positioned above a microfluidics chamber designed to immobilize a worm crawling through the tapered trap. With a worm in place, the electrodes press into the body wall and are capable of recording electrical traces for extended periods of time. During trials with wild-type worms, several cells could be reliably recorded over thirty minute stretches, and two stayed put for nearly an hour.

Depending on how the arrays are built, multiple electrodes can be positioned against a given worm, and multiple worms can be tested on a single platform, provided the animals cooperate in entering and remaining in their chambers. Throughput is up accordingly - the team nearly doubled the daily number of worms previously reported using patch-clamp techniques. Since the animals emerge unscathed after recordings, the platform also opens the possibility of tracking the cells in same animal over time.

"It's just a really high versatility tool," says first author Daniel Gonzales, one that he hopes will make electrophysiology in small invertebrates much more available to others. "There's a lot more that you can do with this tool than you can do with conventional techniques."
The second generation of nanoSPEAR technology is already in the works, centered around improving the signal-to-noise ratio of the recordings. "You'll notice the amplitude of the spikes are not very large compared to the background noise level. They're large enough for us to count and they're large enough for us to get average waveforms," Robinson says, "But we can't get much information about the waveform from an individual measurement." Better immobilization of the animals will be key, with some preliminary data showing improved ratios using a microfluidic valve that holds the worm in place, says Gonzales.

The lab has also been working on combining nanoSPEARs with different imaging techniques and chemical stimulation. Robinson explains, "We can think about using the nanoSPEARs to measure muscle activity while we simultaneously image neural activity using a calcium sensitive indicator or a voltage sensitive fluorescent protein."

Although not quite a 'DIY' technology-a certain level of specialized nanofabrication skill is needed to manufacture the electrodes and platform-both Robinson and Gonzales look forward to future scientific collaborations. Co-author Weiwei Zhong is already putting the platform to use studying different $C$. elegans disease models and others have expressed interest in taking advantage of the new technology's potential. With generation 2.0 just around the corner, Gonzales says, "We feel comfortable making those collaborations and taking that data."

In addition to developing the technology, Robinson sees interesting scientific applications for nanoSPEARs. An obvious direction is to study different neurodegenerative diseases, with an eye towards drug discovery. "I think the idea of being able to phenotype based on electrical activity is actually one of the most useful things," he says, especially as they continue to refine and improve the recordings. The results published in Nature Nanotechnology include what they believe to be the first electrophysiological phenotypes for Parkinson's and ALS worm models.

There are also other invertebrates to interrogate. "In theory, there's not really anything stopping you from trying," says Gonzales,
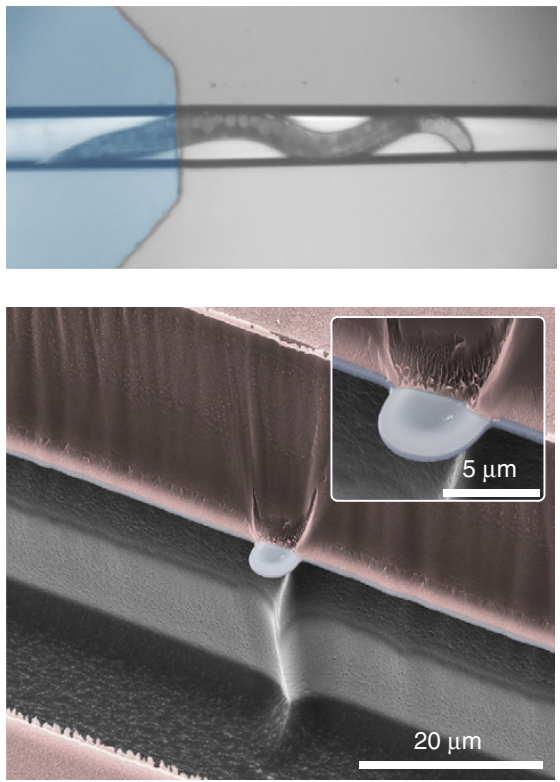

(Top) Optical micrograph of $C$. elegans flowing from the microfluidic layer (blue), into the recording chamber. (Bottom) False-colored scanning electron micrograph of a representative nano-SPEAR. Adapted from Nat. Nanotechnol. 10.1038/nnano.2017.55.

explaining that the size and shape of the chambers could be tailored for different organisms while using the same nanofabrication process. Robinson was introduced to Hydra by Rafael Yuste's lab at Columbia University, and has since been working to transition the platform to accommodate the more delicate cnidarian. With support from DARPA's Biological Control program, Robinson and Yuste are interested in understanding how to re-engineer the nervous system of Hydra. "NanoSPEARs will play a role in helping us track the electrical activity of the animal over time," Robinson says.

Meanwhile, Gonzales, who shifted from the world of physics to develop nanotechnologies as part of his doctoral research at Rice, has been sold on C. elegans. "I'm particularly interested in looking at the motor circuit," he says. "A big question is simply, how do these worms make these crawling motions?" Freed from the confines of patch-clamping, he is excited to test different locomotion theories with the platform he helped develop.

Ellen P. Neff 